\title{
ChemComm
}

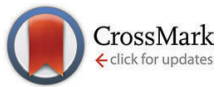

Cite this: Chem. Commun., 2016, 52, 13963

Received 23rd October 2016, Accepted 9th November 2016

DOI: $10.1039 / c 6 c c 08535 k$

www.rsc.org/chemcomm

Nucleophilic ${ }^{18} \mathrm{~F}$-fluorination of bromodifluoromethyl derivatives was performed using $\left[{ }^{18} \mathrm{~F}\right] \mathrm{Bu}_{4} \mathrm{NF}$ in the presence of DBU (1,8-diazabicyclo[5.4.0]undec-7-ene). This novel procedure provided a diverse set of $\left[{ }^{18} \mathrm{~F}\right]$ trifluoroacetamides in good to excellent radiochemical conversions. A mechanism where DBU acts as organomediator in this transformation is proposed.

Due to the favourable properties of fluorine, approximately one fifth of newly registered small drug molecules are organofluorine compounds. ${ }^{1}$ Moreover, the artificial isotope ${ }^{18} \mathrm{~F}\left(t_{1 / 2} 109.8 \mathrm{~min}\right)$ has suitable nuclear properties for in vivo imaging in human subjects using positron emission tomography (PET). ${ }^{2}$ Hence, there is a large demand for efficient fluorination methodologies in both medicinal ${ }^{3}$ and radiosynthetic chemistry. ${ }^{4}$

Despite the remarkable developments in PET radiochemistry during the past decade, ${ }^{4}$ lack of efficient radiochemical methodology may still be a bottleneck in drug discovery and development. It is particularly rate-limiting for microdosing studies, in which the distribution of the radiolabeled drug molecule, previously developed without the intent to be radiolabeled, is studied during the PET measurement. ${ }^{5}$ In view of our long-term objective to facilitate PET studies during drug discovery and development, we turned our attention to the development of a new method for introducing fluorine-18 into trifluoroacetamide $\left(-\mathrm{NCOCF}_{3}\right)$ groups. Although these groups are important motifs in medicinal chemistry, ${ }^{6}$ their radiolabelling has so far been remarkably neglected in the literature. On the other hand, more attention has been directed to the

\footnotetext{
${ }^{a}$ AstraZeneca Personalised Healthcare and Biomarkers, PET Centre at Karolinska Institutet, Karolinska Universitetssjukhuset Solna, R5:02, SE-171 76 Stockholm, Sweden. E-mail: Magnus.Schou@astrazeneca.com ${ }^{b}$ Department of Organic Chemistry, Stockholm University, Arrhenius Laboratory, SE-106 91 Stockholm, Sweden

${ }^{c}$ Stockholm Brain Institute, Karolinska Institutet, SE-171 77 Stockholm, Sweden

${ }^{d}$ CVMD iMed, Medicinal Chemistry AstraZeneca R\&D, Mölndal, SE-431 83, Sweden

${ }^{e}$ Department of Clinical Neuroscience, Karolinska Institutet, S-17176 Stockholm, Sweden

$\dagger$ Electronic supplementary information (ESI) available: Experimental procedures, characterization, radiochemistry and spectral data. See DOI: 10.1039/c6cc08535k
}

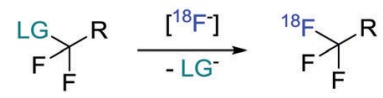

Scheme 1 General synthesis of ${ }^{18} \mathrm{~F}-\mathrm{CF}_{3}$ moieties by displacement of a leaving group.

development of methods for the installation of $\left[{ }^{18} \mathrm{~F}^{-} \mathrm{CF}_{3}\right.$ groups into other type of motifs and two distinct approaches have been taken for this purpose. The first proceeds via direct substitution of a leaving group in the appropriate difluoromethyl analog with ${ }^{18}$ F-fluoride (Scheme 1). ${ }^{7-12}$ Traditionally, these transformations have been thermally activated, but more recently metal salts were successfully used as activators. ${ }^{11}$ The second approach comprises a two-step procedure in which the $\left[{ }^{18} \mathrm{~F}^{2} \mathrm{CF}_{3}\right.$ functionality is incorporated into aromatic substrates via $\left[{ }^{18} \mathrm{~F}\right] \mathrm{CuCF}_{3}$ species. ${ }^{8-10,13}$

We started investigating the transformation of difluorobromoacetamide 1a into the corresponding ${ }^{18} \mathrm{~F}$-trifluoromethyl analog $\left[{ }^{18} \mathrm{~F}\right] 2 \mathrm{a}$ using $\left[{ }^{18} \mathrm{~F}\right] \mathrm{Bu}_{4} \mathrm{NF}$ in a set of solvents with varying temperatures (Table 1, entries 1-5). The reaction proceeded with low to modest radiochemical conversion (RCC; the fraction of radioactivity incorporated into the desired product) in all examined solvents, including DCE (entry 1) acetonitrile (entry 2) and DMSO (entry 3). The highest RCC (35\%) was observed in DMF. There was no obvious difference between kryptofix $/ \mathrm{K}^{18} \mathrm{~F}$ and $\left[{ }^{18} \mathrm{~F}\right] \mathrm{Bu}_{4} \mathrm{NF}$ as the fluorinating species (entries 5 vs. 4). Interestingly, the use of metal salts as additives did not lead to improvements in the RCC either (entries 6-8). In light of these results where reported methods failed, we turned our attention to nitrogen based nucleophilic organic activators for the ${ }^{18} \mathrm{~F}$-fluorination. ${ }^{14}$ Thus, the transformation of $1 \mathrm{a}$ into $\left[{ }^{18} \mathrm{~F}\right] 2 \mathrm{a}$ was investigated in DMF at $100{ }^{\circ} \mathrm{C}$ using 1 equiv. of activator. Though DABCO and DBN did not provide an improvement in RCC (entries 9 and 12 vs. 4), gratifyingly, DMAP and pyridine increased the RCC to 59\% and $68 \%$, respectively (entries 10 and 11). A further increase in RCC to $71 \%$, twice compared to control conditions without activator ( $c f$. entries 4 and 13), was obtained using DBU. When two further guanidine derivatives were used, TBD and MTBD, an even higher RCC was observed (entries 15 and 16, respectively). However, when 
Table $1{ }^{18} \mathrm{~F}$-labelling of $\mathbf{2} \mathbf{a}$ under various conditions ${ }^{a}$

\begin{tabular}{lllll}
\hline & & & \\
& & &
\end{tabular}

the reactions with TBD and MTBD were repeated in the cold lab (using ${ }^{19}$ F-containing TBAF) extensive hydrodebromination of the starting material 1a was also detected. Because formation of this by-product would complicate the purification and isolation of $\left[{ }^{18} \mathrm{~F}\right] 2 \mathrm{a}$ and since the reaction with DBU proceeded with a high RCC without hydrodebromination (or other side reactions), DBU was selected as the activator in the further studies.

With the improved conditions in hand, we explored the scope of the DBU-mediated reaction with different tertiary (1a-1o) and secondary (1p and 1r) bromodifluoroacetamides (Scheme 2). Substrates with cyclic or linear alkyl chains (1a-1b), or including alkene functionalities (1c), gave the labelled amides $\left[{ }^{18} \mathrm{~F}\right] \mathbf{2 a - 2 c}$ in good to excellent RCC (71, 68 and $84 \%$, respectively). The benzylic derivatives 1d-1f and the morpholine, oxetane, proline and ketal derivatives $\mathbf{1 g} \mathbf{- 1 j}$ could also be conveniently transformed with $\left[{ }^{18} \mathrm{~F}\right] \mathrm{TBAF}$ in the presence of DBU to $\left[{ }^{18} \mathrm{~F}\right] \mathbf{2 d}-\mathbf{2} \mathbf{j}$ (61-92\% RCC). In metal catalyzed/mediated reactions, sulfur has a tendency to coordinate to the metal atom and thus inhibit the reaction. With the current methodology, thioether derivative $\left[{ }^{18} \mathrm{~F}\right] \mathbf{2 k}$ was obtained in excellent RCC (84\%), thus suggesting an apparent insensitivity of the protocol to thiol functionalities. Next, substrate 1l, with an additional amide function, and 1m-1o, with some important nitrogen containing heterocycles commonly used in medicinal chemistry, were converted smoothly into trifluoromethyl derivatives $\left[{ }^{18} \mathrm{~F}\right] \mathbf{2 1 - 2 0}$ in excellent RCCs (77, 90, 75 and 80\%, respectively). Finally, we focused our attention to the more challenging secondary bromodifluoroacetamide substrates $1 \mathbf{p}$ and 1r. As expected, $\left[{ }^{18} \mathrm{~F}\right] 2 \mathbf{p}$ and $\left[{ }^{18} \mathrm{~F}\right] 2 \mathbf{r}$ were obtained in substantially lower RCCs than the tertiary amide derivatives using direct DBU-mediated fluorination. Considering that the

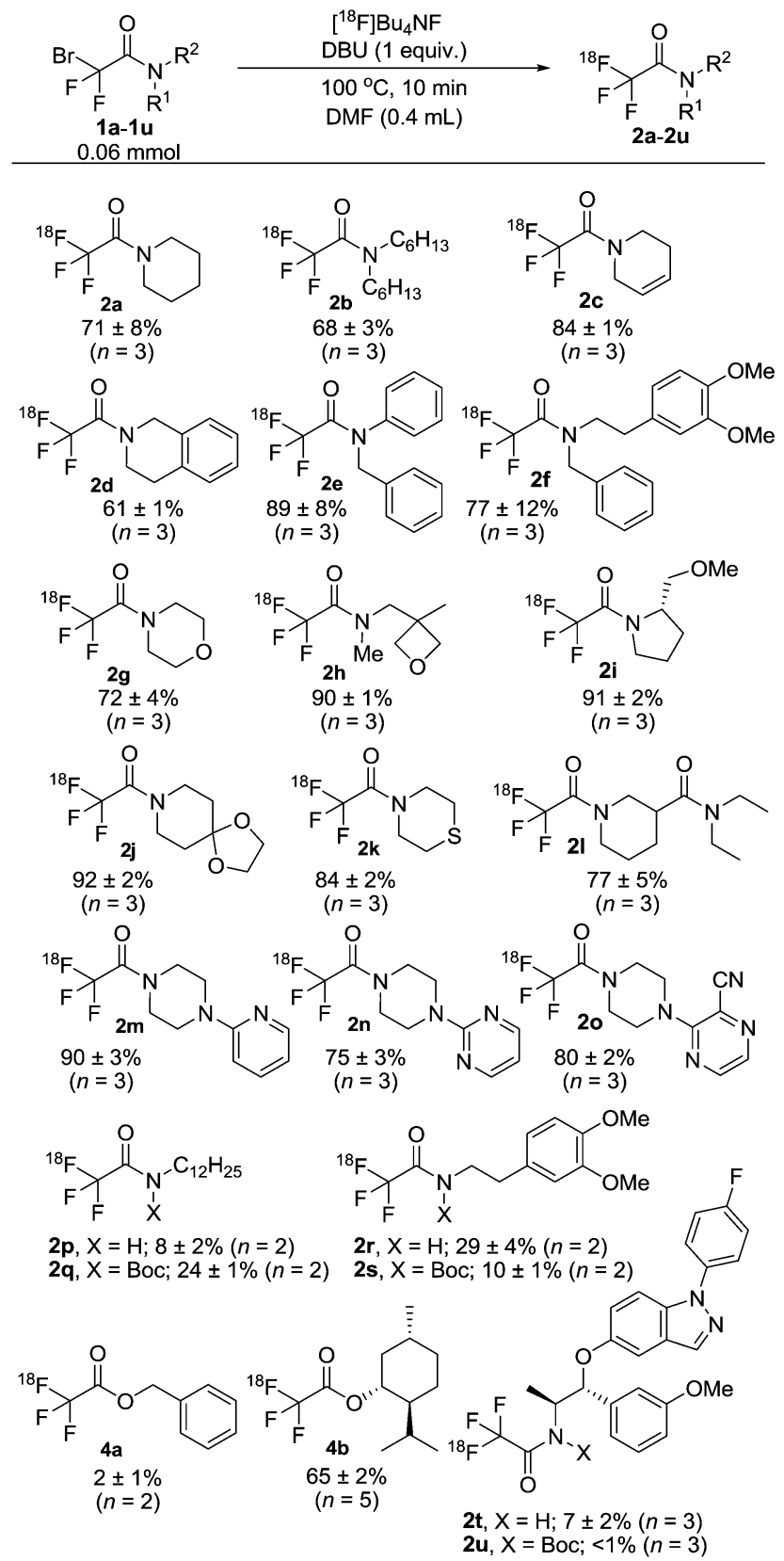

Scheme 2 Substrate scope in the ${ }^{18} \mathrm{~F}$-labelling mediated by DBU. $\mathbf{1 a - 1 u}$ : bromodifluoroacetamides, $\mathbf{2 a - 2} \mathbf{u}$ : trifluoroacetamides. RCCs estimated by radio-HPLC. Boc = tert-butoxycarbonyl.

introduction of a benzyl group into 1r provided a threefold increase in RCC $\left(c f .\left[{ }^{18} \mathrm{~F}\right] 2 \mathbf{f} v s .\left[{ }^{18} \mathrm{~F}\right] 2 \mathbf{r}\right)$ we decided to explore a protecting group strategy for the secondary amide substrates. However, much to our disappointment, $N$-Boc (tert-butoxycarbonyl) protection was not a viable solution for this purpose as it provided only marginal improvement, or even a reduction, in the RCC for $\left[{ }^{18} \mathrm{~F}\right] 2 \mathbf{q}$ and $\left[{ }^{18} \mathrm{~F}\right] \mathbf{2 s}$.

In an effort to improve the yields for this important class of compounds, the protocol was modified to include an ${ }^{18} \mathrm{~F}$-labelled ester as intermediate (Scheme 3). ${ }^{15}$ Because of the basic radiofluorination reaction media, extensive hydrolysis was observed during the synthesis of ester $\left[{ }^{18} \mathrm{~F}\right] \mathbf{4 a}$ and the resulting RCC 


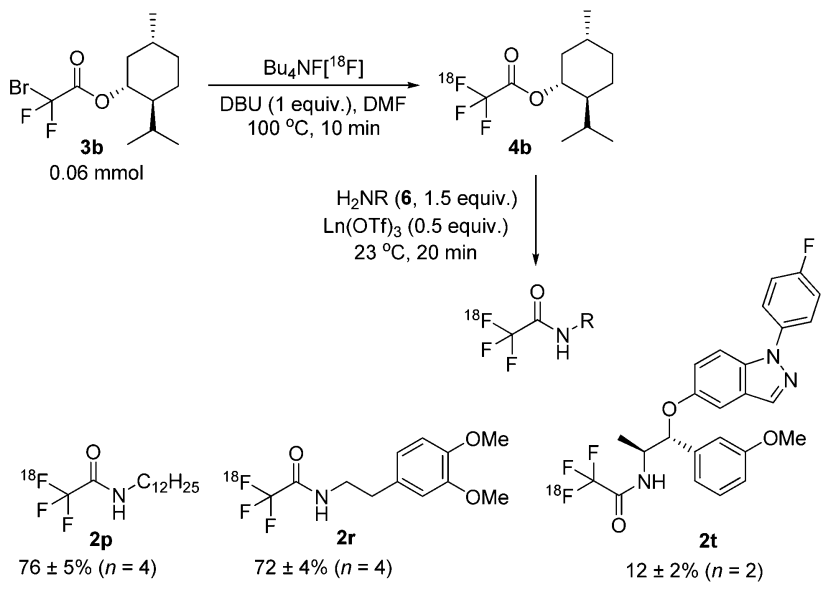

Scheme 3 Radiolabelling of $\left[{ }^{18} \mathrm{~F}\right] \mathbf{4 b}$ and derivatization to secondary amides. RCCs were estimated by radio-HPLC.

was only $2 \%$. Gratifyingly, however, the more stable menthol ester $\left[{ }^{18} \mathrm{~F}\right] \mathbf{4 b}$ could be obtained in a $64 \%$ RCC and further converted, without intermediate purification, into the desired secondary amides $\left[{ }^{18} \mathrm{~F}\right] 2 \mathbf{p}$ and $\left[{ }^{18} \mathrm{~F}\right] \mathbf{2 r}$ with high RCCs (76\% and $72 \%$ respectively). To evaluate the utility of the current methodology for the labelling of druglike molecules it was applied to the ${ }^{18} \mathrm{~F}$-labelling of $\left[{ }^{18} \mathrm{~F}\right] \mathbf{2 t}$ (AZD5423), a nonsteroidal glucocorticoid agonist developed for the treatment of respiratory diseases. ${ }^{12,16}$ Because $\left[{ }^{18} \mathrm{~F}\right] 2 \mathrm{t}$ is a secondary amide, direct labelling in the presence of DBU proceeded with only a modest RCC (7\%, Scheme 2) and starting from the $N$-Boc protected derivative 1u failed. However, when using the above sequence (Scheme 3), with $\left[{ }^{18} \mathrm{~F}\right] \mathbf{4 b}$ as a key intermediate, we obtained the target molecule in a $12 \%$ RCC under non-optimized conditions.

In a preparative run, starting from $762 \mathrm{MBq}$ of $\left[{ }^{18} \mathrm{~F}\right] \mathrm{Bu}_{4} \mathrm{NF}$, $\left[{ }^{18} \mathrm{~F}\right] 2 \mathrm{a}$ was isolated by semi-preparative HPLC in a $44 \%$ radiochemical yield (RCY, not corrected for decay) with a specific activity (S.A. = radioactivity per molar amount of product) of $0.10 \mathrm{GBq}_{\mu \mathrm{mol}}{ }^{-1}$. Although an S.A. of $0.1 \mathrm{GBq} \mu \mathrm{mol}^{-1}$ is useful for PET microdosing studies, it is insufficient for examining drug-target engagement. ${ }^{17}$ Accordingly, a more general application of the methodology for PET studies require an increase of the S.A of the product. In control experiments when $\left[{ }^{18} \mathrm{~F}\right] \mathrm{Bu}_{4} \mathrm{NF}$ was omitted from the reaction mixture, it was found that the formation of $\left[{ }^{19} \mathrm{~F}\right] \mathbf{2 a}$ was largely dependent on the amount of $\mathbf{1 a}$ used in the reaction. ${ }^{9}$ This indicates that ${ }^{19} \mathrm{~F}$ from the $\mathrm{CF}_{2} \mathrm{Br}$ group of $\mathbf{1 a}$ assists in the formation of $\left[{ }^{19} \mathrm{~F}\right] 2 \mathrm{a}$, which lowers the S.A. of the product. This problem can be circumvented by lowering the concentration of $\mathbf{1 a}$ in the reaction mixture. Indeed, by a 100 -fold reduction in the amount of $1 \mathrm{a}$ (from 60 to $0.6 \mu \mathrm{mol}$, see $\mathrm{ESI} \dagger$ ) and starting from $7.16 \mathrm{GBq}$ of $\left[{ }^{18} \mathrm{~F}\right] \mathrm{Bu}_{4} \mathrm{NF},\left[{ }^{18} \mathrm{~F}\right] 2 \mathrm{a}(284 \mathrm{MBq}, 4 \% \mathrm{RCY})$ was obtained with an 84-fold improved specific radioactivity (8.4 $\left.\mathrm{GBq} \mu \mathrm{mol}^{-1}\right)$. Compared to literature examples, ${ }^{7,11}$ this is a decent level of S.A. for a $\left[{ }^{18} \mathrm{~F}\right] \mathrm{CF}_{3}$ species, which inherently suffers from low S.A. Furthermore, since we observed that the formation of cold 2a was unaffected by the radioactivity used in the reaction, the S.A. of $\left[{ }^{18} \mathrm{~F}\right] 2 \mathrm{a}$ is expected to be proportional also to the starting radioactivity. Thus, we envisage that the S.A. could be easily increased further under automated conditions when a full cyclotron production may be used for the radiofluorination. ${ }^{18}$ This would provide a product with a S.A. useful also for drugtarget engagement studies. According to our studies neither the RCY nor the S.A. can be increased by increasing the amount of DBU ( 1 equiv.) in the reaction mixture.

A very interesting feature of the above study is the acceleration effect of DBU in the bromide ${ }^{18} \mathrm{~F}$ exchange process ( $c f$. Table 1 entries 4 and 13). In order to rationalize this effect, we monitored the reaction of $1 \mathrm{a}$ with $\mathrm{DBU}$ using ${ }^{13} \mathrm{C}$ NMR spectroscopy (Scheme 4). The ${ }^{13} \mathrm{C}$ NMR spectra of DBU and isolated 1a are given in Scheme $4 \mathrm{~b}$ and c. When 1a and DBU was mixed in DMF- $\mathrm{d}_{7}$ and heated at $100{ }^{\circ} \mathrm{C}$ (simulating the reaction conditions according to Table 1 , entry 4 ), the ${ }^{13} \mathrm{C}$ NMR signals of DBU underwent systematic changes (Scheme 4a). The signal at $46.5 \mathrm{ppm}$ (assigned to C11) was shifted to $40.5 \mathrm{ppm}$ and substantially broadened. The ${ }^{13} \mathrm{C}$ signal at $38.1 \mathrm{ppm}$ (assigned to C6) also underwent a similar change shifting to $33.6 \mathrm{ppm}$. The shift of the ${ }^{13} \mathrm{C}$ NMR signals clearly shows that chemical environment of the above carbon atoms in DBU is significantly changed on addition of 1a. Most probably DBU displaces the bromide of 1a to form intermediate 7 (Scheme 4). The broadening of the shifts at 40.0 and $33.6 \mathrm{ppm}$ may indicate $\mathrm{C}-\mathrm{F}$ coupling and/or hindered rotation along the $\mathrm{C}\left(\mathrm{F}_{2}\right)-\mathrm{N}$ bond in 7 . The ${ }^{13} \mathrm{C}$ NMR spectrum of the mixture of DBU $+\mathbf{1 a}$ (Scheme $4 \mathrm{a}$ ) remained unchanged in the temperature range of $85-100{ }^{\circ} \mathrm{C}$. When the reaction mixture of $\mathrm{DBU}+\mathbf{1 a}$ was cooled to room temperature intermediate 7 decomposed to DBU and 1a. This indicates that formation of 7 is reversible ${ }^{14}$ and its formation requires heating to at least to $85{ }^{\circ} \mathrm{C}$. Based on the above, we suggest that $\mathrm{DBU}$ and $\mathrm{CF}_{2} \mathrm{Br}$ precursors 1 form intermediates such as 7 prior to the reaction with $\left[{ }^{18} \mathrm{~F}\right] \mathrm{TBAF}$. In 7 two quaternary centers forms a strained $\mathrm{C}\left(\mathrm{F}_{2}\right)-\mathrm{N}$ bond, which is probably easier to cleave than the $\mathrm{C}\left(\mathrm{F}_{2}\right)-\mathrm{Br}$ bond. The behaviour of DBU as a nucleophilic catalyst is known in the literature. ${ }^{14}$ However, this is the first study, when DBU is applied for mediating

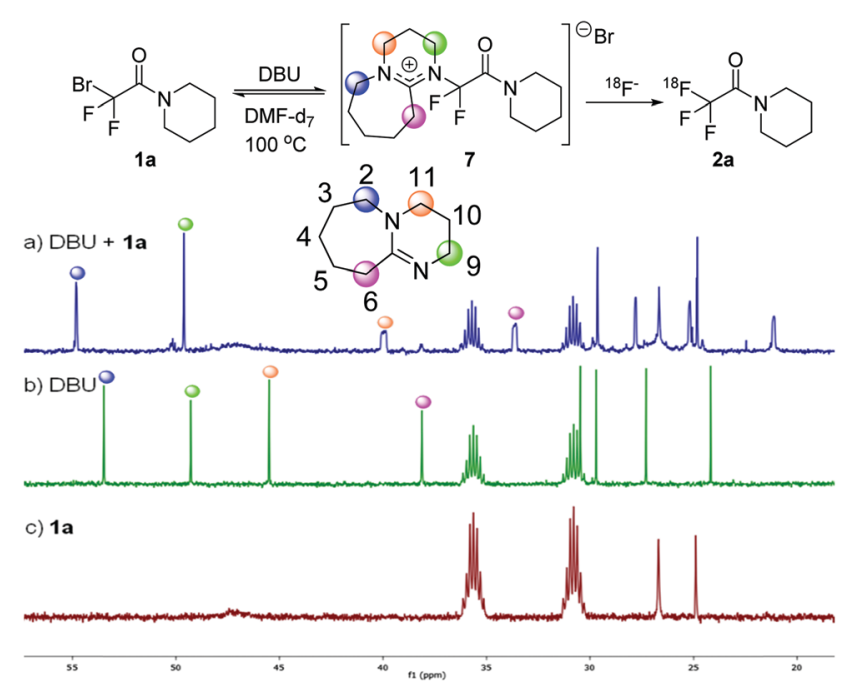

Scheme $4{ }^{13} \mathrm{C}$ NMR studies for monitoring the formation of intermediate 7. ${ }^{13} \mathrm{C}$ NMR spectra $(125 \mathrm{MHz})$ at $100{ }^{\circ} \mathrm{C}$ in $\mathrm{DMF}-\mathrm{d}_{7}$. (a) $\mathrm{DBU}+\mathbf{1 a}$. (b) DBU. (c) $1 \mathrm{a}$ 
the displacement of bromide from $\mathrm{CF}_{2} \mathrm{Br}$ group and application for ${ }^{18} \mathrm{~F}$ labelling.

In summary, we have shown for the first time that ${ }^{18} \mathrm{~F}$-labelled trifluoroacetamides can be efficiently synthesized from easily accessible $\mathrm{CF}_{2} \mathrm{Br}$ derivatives and $\left[{ }^{18} \mathrm{~F}\right] \mathrm{TBAF}$ in the presence of DBU (or analogues). The ${ }^{18}$ F-labelled tertiary trifluoroacetamide products are typically formed with high RCC. For ${ }^{18}$ F-labelling of secondary trifluoroacetamides, we propose a modified work-flow via synthesis of an ${ }^{18} \mathrm{~F}$-labelled ester reagent, which undergo transesterification with the corresponding amine. The radiosynthetic utility of the method was demonstrated by ${ }^{18} \mathrm{~F}$-labelling of the druglike substance $\left[{ }^{18} \mathrm{~F}\right] 2 \mathrm{t}$ (AZD5423). The preparative scale experiments show that this method is suitable to produce ${ }^{18} \mathrm{~F}$-labelled trifluoroacetamides with sufficiently high specific activity for PET microdosing studies and with slight modification even for drug-target engagement studies $\left(8.4 \mathrm{GBq}_{\mu \mathrm{mol}}^{-1}\right.$ for $\left.\left[{ }^{18} \mathrm{~F}\right] 2 \mathrm{2a}\right)$. Mechanistic studies suggest that $\mathrm{DBU}$ has an activating effect on the displacement of bromide from the $\mathrm{CF}_{2} \mathrm{Br}$ group of the precursor 1 . This study broadens the radiosynthetic scope of $\left[{ }^{18} \mathrm{~F}\right] \mathrm{CF}_{3}$ derivatives and assists in the development of new bromide to ${ }^{18} \mathrm{~F}$ exchange methods where thermal activation or metal mediated transformations are unsuccessful.

The authors like to thank the members of the PET group at Karolinska Institutet. Support from AstraZeneca, Stockholm Brain Institute, the Swedish Research Council, the Knut and Alice Wallenberg Foundation are gratefully acknowledged. A. B. G. is grateful to the AstraZeneca Postdoctoral Programme for funding a postdoctoral contract.

\section{Notes and references}

1 (a) K. Müller, C. Faeh and F. Diederich, Science, 2007, 317, 1881; (b) J. Wang, M. Sánchez-Roselló, J. L. Aceña, C. del Pozo, A. E. Sorochinsky, S. Fustero, V. A. Soloshonok and H. Liu, Chem. Rev., 2014, 114, 2432; (c) Y. Zhou, J. Wang, Z. Gu, S. Wang, W. Zhu, J. L. Aceña, V. A. Soloshonok, K. Izawa and H. Liu, Chem. Rev., 2016, 116, 422; (d) H.-J. Böhm, D. Banner, S. Bendels, M. Kansy, B. Kuhn, K. Müller, U. Obst-Sander and M. Stahl, ChemBioChem, 2004, 5, 637; (e) J. Wang, M. Sánchez-Roselló, J. L. Aceña, C. del Pozo, A. E. Sorochinsky, S. Fustero, V. A. Soloshonok and H. Liu, Chem. Rev., 2014, 114, 2432; $(f)$ Y. Zhou, J. Wang, Z. Gu, S. Wang, W. Zhu, J. L. Aceña, V. A. Soloshonok, K. Izawa and H. Liu, Chem. Rev., 2016,
116, 422; $(g)$ H.-J. Böhm, D. Banner, S. Bendels, M. Kansy, B. Kuhn, K. Müller, U. Obst-Sander and M. Stahl, ChemBioChem, 2004, 5, 637.

2 P. W. Miller, N. J. Long, R. Vilar and A. D. Gee, Angew. Chem., Int. Ed., 2008, 47, 8998.

3 (a) J. Charpentier, N. Früh and A. Togni, Chem. Rev., 2015, 115, 650; (b) C. Alonso, E. Martínez de Marigorta, G. Rubiales and F. Palacios, Chem. Rev., 2015, 115, 1847; (c) W. Kong, E. Merino and C. Nevado, Chimia, 2014, 68, 430; (d) J. R. Wolstenhulme and V. Gouverneur, Acc. Chem. Res., 2014, 47, 3560; (e) T. Liang, C. N. Neumann and T. Ritter, Angew. Chem., Int. Ed., 2013, 52, 8214.

4 (a) K. Chansaenpak, B. Vabre and F. P. Gabbai, Chem. Soc. Rev., 2016, 45, 954; (b) S. Preshlock, M. Tredwell and V. Gouverneur, Chem. Rev., 2016, 116, 719; (c) C. N. Neumann and T. Ritter, Angew. Chem., Int. Ed., 2015, 54, 3216.

5 M. Schou, K. Varnäs, S. Lundquist, R. Nakao, N. Amini, A. Takano, S. J. Finnema, C. Halldin and L. Farde, Int. J. Neuropsychopharmacol., $2015,18,10$

6 (a) K. Edman, R. Ahlgren, M. Bengtsson, H. Bladh, S. Bäckström, J. Dahmén, K. Henriksson, P. Hillertz, V. Hulikal, A. Jerre, L. Kinchin, C. Kåse, M. Lepistö, I. Mile, S. Nilsson, A. Smailagic, J. Taylor, A. Tjörnebo, L. Wissler and T. Hansson, Bioorg. Med. Chem. Lett., 2014, 24, 2571; (b) G. M. Gauvreau, L.-P. Boulet, R. Leigh, D. W. Cockcroft, K. J. Killian, B. E. Davis, F. Deschesnes, R. M. Watson, V. Swystun, C. K. Mårdh, P. Wessman, C. Jorup, M. Aurivillius and P. M. O'Byrne, Am. J. Respir. Crit. Care Med., 2015, 191, 161.

7 V. T. Lien and P. J. Riss, BioMed Res. Int., 2014, 2014, 10.

8 T. Rühl, W. Rafique, V. T. Lien and P. J. Riss, Chem. Commun., 2014, 50, 6056.

9 M. Huiban, M. Tredwell, S. Mizuta, Z. Wan, X. Zhang, T. L. Collier, V. Gouverneur and J. Passchier, Nat. Chem., 2013, 5, 941.

10 D. van der Born, C. Sewing, J. D. M. Herscheid, A. D. Windhorst, R. V. A. Orru and D. J. Vugts, Angew. Chem., Int. Ed., 2014, 53, 11046.

11 (a) T. Khotavivattana, S. Verhoog, M. Tredwell, L. Pfeifer, S. Calderwood, K. Wheelhouse, T. Lee Collier and V. Gouverneur, Angew. Chem., Int. Ed., 2015, 54, 9991; (b) S. Verhoog, L. Pfeifer, T. Khotavivattana, S. Calderwood, T. L. Collier, K. Wheelhouse, M. Tredwell and V. Gouverneur, Synlett, 2016, 25.

12 P. Johnstrom and S. Stone-Elanderl, J. Labelled Compd. Radiopharm., 1995, 36, 537.

13 P. Ivashkin, G. Lemonnier, J. Cousin, V. Grégoire, D. Labar, P. Jubault and X. Pannecoucke, Chem. - Eur. J., 2014, 20, 9514.

14 J. E. Taylor, S. D. Bull and J. M. J. Williams, Chem. Soc. Rev., 2012, 41, 2109. 15 (a) H. Morimoto, R. Fujiwara, Y. Shimizu, K. Morisaki and T. Ohshima, Org. Lett., 2014, 16, 2018; (b) L. Wang, X.-J. Wei, W.-L. Jia, J.-J. Zhong, L.-Z. Wu and Q. Liu, Org. Lett., 2014, 16, 5842.

16 (a) M. Berger, D. Jan, A. Eriksson, B. Gabos, T. Hanson, M. Hemmerling, K. Henriksson, S. Ivanova, M. Lepisto, D. McKerrecher, M. Munck af Rosenschold, S. Nilsson, H. Rehwinkel and C. Taflin, WO2008/076048, 2008; (b) T. Eriksson and T. Hansson, WO2010/008341A1, 2010.

17 S. E. Lapi and M. J. Welch, Nucl. Med. Biol., 2013, 40, 314.

18 H. Shi, A. Braun, L. Wang, S. H. Liang, N. Vasdev and T. Ritter, Angew. Chem., Int. Ed., 2016, 55, 10786. 